12

\title{
Clinical presentation and management of hypoparathyroidism
}

\author{
Gemma Marcucci, MD, PhD, Assistant Professor of \\ Endocrinology, \\ Luisella Cianferotti, MD, PhD, Assistant Professor of \\ Endocrinology, \\ Maria Luisa Brandi, MD, PhD, Professor of Endocrinology *
}

Department of Surgery and Translational Medicine, University of Florence, Bone Metabolic Diseases Unit, University Hospital of Florence, Italy

\section{A R T I C L E I N}

\section{Article history:}

Available online 28 September 2018

\section{Keywords:}

hypoparathyroidism

hypocalcemia

symptomatology
The clinical manifestations of hypoparathyroidism are variable and can involve almost any organ system. The main clinical features of the hypoparathyroidism are typically signs or symptoms due to neuromuscular irritability owing to low serum calcium level. In addition to hypocalcemia, hyperphosphatemia can contribute to long-term complications, including extra-skeletal calcifications. Bone turnover markers are generally decreased, and bone mass density is usually normal-increased compared to age- and gendermatched controls. It is still unclear whether or not these bone features could have an impact on the risk of fracture. Impaired renal function is a common complication described in patients treated for hypoparathyroidism. Other complications include premature cataracts, seizures, basal ganglia calcifications, and cardiac arrhythmias. Lastly, some clinical studies have also reported a reduced quality of life of patients with hypoparathyroidism. Increased awareness of the clinical manifestations of this disease is important to improve its clinical management.

(C) 2018 Elsevier Ltd. All rights reserved.

\footnotetext{
* Corresponding author. Department of Surgery and Translational Medicine, University of Florence, Largo Palagi 1, 50139, Florence, Italy. Fax: +39 0557946303.

E-mail addresses: gemma.marcucci@unifi.it (G. Marcucci), luisella.cianferotti@unifi.it (L. Cianferotti), marialuisa.brandi@ unifi.it (M.L. Brandi).
} 


\section{Introduction}

The clinical manifestations of hypoparathyroidism are several and variable involving most organ system. Low serum ionized calcium level can impair several organic functions, such as neurological, cognitive, muscular, and cardiac functions [1-4]. Neuromuscular irritability due to hypocalcemia is the typical symptom of this disorder.

In hypoparathyroidism, the hypocalcemia can present emergently in case of anterior neck surgery or supplemental calcium and active vitamin change or who are noncompliant with treatment. Acute hypocalcemia states represent a medical emergency and potentially life-threatening, and the pharmacologic intervention should be timely. In case of chronic hypocalcemia, the clinical manifestations can be mild and often nonspecific neuromuscular symptoms. Sometime, the biochemical screening test can reveal a status of hypocalcemia due to hypoparathyroidism not associate to severe symptomatology [5]. Chronic high phosphate levels and high calcium-phosphate product, resulting from the disease itself and mostly the treatment with calcium and calcitriol, or other active vitamin D analogs supplements, have an important role in the development of the ectopic calcifications [1]. The typical sites of extraskeletal calcifications are: the brain (basal ganglia in particular), kidney, or joints, eyes, skin, vasculature, and other organ systems [2-7]. In addition to the alterations of calcium and phosphate, low serum magnesium levels can be found in these patients, and the magnesium deficit can aggravate the signs and symptoms due to hypocalcemia [1]. The pathogenesis of other clinical features, instead, is not yet currently well known, such as neuropsychiatric symptoms [8,9]. Table 1 summarizes the main clinical manifestations of chronic hypoparathyroidism. The effects of hormone replacement therapy with PTH peptides on clinical manifestations and complications are described in the review regarding the treatment of hypoparathyroidism, included in this monothematic issue.

\section{Biochemical features}

The hypoparathyroidism is typically characterized by low serum albumin-corrected calcium or ionized calcium (below the lower limit of the normal range; respectively $<8.5 \mathrm{mg} / \mathrm{dl}$ or $2.12 \mathrm{mmol} / \mathrm{l}$ )

Table 1

Clinical manifestations of chronic hypoparathyroidism.

\begin{tabular}{|c|c|}
\hline Organ/system & Clinical manifestation (signs and symptoms) \\
\hline Neuromuscular & $\begin{array}{l}\text { Tetany, muscle cramping (carpal and/or pedal spams) and twitching (Chvostek's sign,Trousseau's sign). } \\
\text { Laryngospasm and stridor, bronchospasm and wheezing. Fatigue, muscle weakness. }\end{array}$ \\
\hline Neurological & $\begin{array}{l}\text { Central nervous system involvement: seizures; basal ganglia and brain calcifications (Fahr's disease), } \\
\text { parkinsonism or dystonia, altered mental status, impaired memory and concentration, pseudotumor } \\
\text { cerebri, extrapyramidal disorders, chorea. } \\
\text { Peripheral nervous system involvement: paresthesia and numbness (i.e. around the mouth, in the fingers, } \\
\text { toes and circumoral region). }\end{array}$ \\
\hline Psychiatric & Depression, anxiety, personality disturbances. \\
\hline Kidney & $\begin{array}{l}\text { Hypercalciuria, ectopic mineralization in the kidney (renal stones, nephrocalcinosis). } \\
\text { Renal function: decreased glomerular filtration rate, kidney failure. }\end{array}$ \\
\hline Musculoskeletal & Myopathy, elevated creatinine kinase. Normal-high BMD. \\
\hline Cardiovascular & $\begin{array}{l}\text { Cardiac involvement: congestive heart failure (cardiomegaly, pulmonary congestion, volume overload), } \\
\text { chest pain, heart block. } \\
\text { ECG alterations: cardiac arrhythmias, prolonged QTc interval, QRS and ST segment changes suggestive of } \\
\text { myocardial ischemia/infarction, ventricular arrhythmias. }\end{array}$ \\
\hline Respiratory & Laryngospasm, bronchospasm. \\
\hline Ophthalmological & Papilledema, cataracts (posterior subcapsular cataracts), calcification of the cornea. \\
\hline Dermatological & $\begin{array}{l}\text { Alopecia, scaling of the skin, deformities of the nails, onycholysis, dry skin, coarse, thin hair, pustular } \\
\text { psoriasis. }\end{array}$ \\
\hline Gastrointestinal & Constipation, abdominal cramps, steatorrhea. \\
\hline Dental & $\begin{array}{l}\text { Altered tooth morphology, cemental hyperplasia, abnormal dentition (enamel hypoplasia), short } \\
\text { rounded roots, hypodontia and delay or lack of tooth eruption, possibly widening of the periodontal } \\
\text { ligament space. }\end{array}$ \\
\hline
\end{tabular}

Abbreviation: ECG: electrocardiogram.

References: [1-3,5,8,10]. 
and 1,25- dihydroxyvitamin $\mathrm{D}\left[1,25(\mathrm{OH})_{2} \mathrm{D}\right]$ levels, increased serum phosphorus concentration, and low or inappropriate, for the degree of hypocalcemia, intact parathyroid hormone (PTH) levels [3]. Since, about $50 \%$ of the total serum calcium is represented by the ionized fraction, and the remainder is protein-bound or complexed to anions, in evaluating the degree of hypocalcemia, the corrected serum calcium should be used, according to the following formula: corrected serum total $\mathrm{Ca}=$ measured total $\mathrm{Ca}+[0.8 \times(4.0$-measured serum albumin) $]$ [5]. The measurement of free or ionized serum calcium should be more accurate than the albumin-corrected total serum calcium concentration and is preferred in pregnancy. However, there are several limitations to the accurate direct measurement of ionized calcium. Sometime, the clinical utility of the ionized calcium measurement is limited by the technical issues $[10,11]$. The 24 -h urine calcium level may be low-normal before calcium and vitamin $\mathrm{d}$ metabolites or analogs supplementation is started, and can be found increased during treatment [3]. Biochemical investigations are useful to differentiate hypoparathyroidism from other diseases characterized by hypocalcemia, such as pseudohypoparathyroidism (PHP), a disorder due to PTH resistance causing increased PTH levels, hypocalcaemia and hyperphosphataemia [12].

A reliable assay for measuring serum PTH is essential for diagnosis of hypoparathyroidism. Fulllength, active PTH (1-84) peptides and several forms of truncated, mostly carboxyl-terminal fragments [especially PTH (34-84) and PTH (37-84)] represent the circulating PTH peptides $[13,14]$. The truncated fragments cannot activate the classic PTH1 receptor (PTH1R). In euparathyroid subjects, the two-site immunoradiometric assay (IRMA) for PTH do not recognize large and inactive mid- and carboxy-terminal fragments of PTH, but detect together the carboxy- and amino-terminal ends of the molecule, that represent most circulating PTH [15-18]. This assay is the most widely used intact PTH assay to date, and provides excellent discrimination between hypoparathyroidism and the hypocalcemic states of secondary hyperparathyroidism, but it not detect the majority of carboxyterminalterminal fragments [8]. Subsequently, a third-generation PTH assay (called also the "whole PTH" or "biointact PTH" assay) was introduced [19]. This assay uses a C-terminal capture antibody and an $\mathrm{N}$-terminal detection antibody detecting only the extreme N-terminal region of PTH [PTH (1-6)]. However, it has not been proven to be superior in clinical practice, although studies are limited $[20,21]$.

Magnesium $\left(\mathrm{Mg}^{2+}\right)$ deficit can be associated with impaired secretion and action of PTH [22]. Then, in case of hypocalcemia without a known etiology, serum magnesium levels should be measured [23]. However, serum $\mathrm{Mg}^{2+}$ is considered a poor marker of the body's $\mathrm{Mg}^{2+}$ stores for estimating total body magnesium content, and more precise methods have been developed (i.e. the erythrocyte $\mathrm{Mg}^{2+}$ concentration and the "magnesium tolerance test"), but for primarily research settings [22]. Causes of hypomagnesemia can be divided into: decreased intake, absorption, or increased losses, and redistribution [22]. Under normal circumstances, low intake of magnesium with the diet is usually not common [24]. The most common causes of $\mathrm{Mg}^{2+}$ decreased absorption include severe diarrhea, especially when due to laxative abuse, steatorrhea, malabsorption syndromes, and short bowel syndrome [22]. Some drugs can determine decreased tubular reabsorption, such as diuretics, antibiotics, calcineurin inhibitors, and epidermal growth factor (EGF) receptor antagonists. Severe levels of hypomagnesemia $\left(\mathrm{Mg}^{2+}\right.$ levels $\left.<0.4 \mathrm{mmol} / \mathrm{L}\right)$ inhibit PTH secretion causing relative hypoparathyroidism, whereas mild decreases in $\mathrm{Mg}^{2+}$ levels to as low as $0.5 \mathrm{mmol} / \mathrm{L}$ stimulates PTH secretion [22]. The intracellular $\mathrm{Mg}^{2+}$ depletion seems to act on the $\alpha$-subunits of the heterotrimeric G-proteins associated with the CaSR inhibiting PTH. Moreover, high serum $\mathrm{Mg}^{2+}$ levels may cause hypocalcemia, although it is usually mild in severity and asymptomatic [22].

Biochemical evaluation should include the measurement of serum concentration of 25hydroxyvitamin D (25OHD), as it may be of importance to a number of cellular processes and may undergo hydroxylation to $1,25(\mathrm{OH})_{2}$ D catalyzed by local hydroxylases in different tissues $[10,25]$.

\section{Renal manifestations}

Physiologically, PTH increases renal tubular calcium reabsorption and urinary phosphate excretion, therefore, in case PTH effects on tubular functions are lacking, hypercalciuria and reduced urinary phosphate excretion would be expected [5]. However, in chronic hypoparathyroidism, urinary calcium and phosphate excretion may be normal in hypoparathyroid subjects not treated with calcium and vitamin D supplements, because the filtered load of calcium tends to be lower compared to normal 
condition, and, high serum phosphate levels determines a greater filtered load of phosphate [5]. Instead, hypercalciuria often is reported in hypoparathyroid subjects treated with large amounts of calcium and/or active vitamin D assumed in order to control of the serum calcium level [5]. High sodium intakes may be associated with hypercalciuria [24].

The elevated calcium-phosphate product and hypercalciuria is associated to the risk of renal stones or calcinosis, especially if large amounts of calcium and active vitamin D are needed [26]. In hypoparathyroid patients treated with calcium and activated vitamin D supplementation, the reported prevalence rates of nephrocalcinosis varies between 12 and 57\% [8,27]. A large Danish case-control study of patients $(\mathrm{n}=688)$ with post-surgical hypoparathyroidism described an increased risk of renal complications (hazard ratio [HR], 3.67; 95\% confidence interval [CI], 2.41-5.59) and of developing kidney stones equal to 4.82 (95\% CI: 2.00-11.64) in patients compared with controls [28].

The chronic standard treatment in these patients may cause also an increased risk of renal function impairment and chronic kidney disease in the long run, particularly in those with activating CaSR mutations $[2,26]$. Impaired renal function has been associated with duration of the disease, the age of the patient, and relative time with hypercalcemia $[10,26]$. These renal complications are the most serious long-term risks for patients with chronic hypoparathyroidism [2,26]. In a US large cohort of patients (n: 120) with permanent hypoparathyroidism, (n:44) $41 \%$ of patients had an estimated glomerular filtration rate (eGFR) of $<60 \mathrm{ml} / \mathrm{min} / 1.73 \mathrm{~m}^{2}$ (normal value: eGFR of $\geq 90 \mathrm{ml} / \mathrm{min} / 1.73 \mathrm{~m}^{2}$ ), consistent with chronic kidney disease stage 3 or higher, which was 2-17-fold higher compared to age-adjusted normal values [26]. A Danish case-control study with patients affected by nonsurgical hypoparathyroidism $(\mathrm{n}=180)$ had a significantly increased risk of renal insufficiency (hazard ratio [HR] 6.01) compared with controls [29]. Lastly, another Danish case-control study with patients affected by postsurgical hypoparathyroidism ( $\mathrm{n}$ : 688) described that the hazard ratios for diagnosed renal insufficiency was 3.10 (95\% CI: $1.73-5.55)$ [28].

\section{Bone manifestations}

Physiologically, in adulthood bone mass is regulated by bone remodeling process, a tightly regulated balance between bone resorption and formation. PTH has a main role in regulation of the rate of bone remodeling, that promotes through its direct actions on osteoblasts and osteocytes and indirect effects on osteoclasts [30]. Deficit or inappropriate level of PTH causes early a decrease in bone resorption and then to a coupled reduction in bone formation. Afterwards, bone mass tends to increase, since the balance between resorption and formation favors the latter [3,5]. It is still controversial, whether these structural and dynamic skeletal abnormalities may affect bone strength or fracture risk in hypoparathyroidism [29,31,32].

Chronic hypoparathyroidism is characterized typically by bone mineral density (BMD), as determined by dual energy x-ray absorptiometry, somewhat greater compared to age- and sex-matched controls [31-36]. Increased BMD has been described in men and women affected by postsurgical, autoimmune, and idiopathic hypoparathyroidism, on long-term treatment with calcium and vitamin $\mathrm{D}$ supplements. Overall, BMD tends to be increased at all skeletal sites, especially at the lumbar spine [37]. Trabecular bone score (TBS), an indirect measure of bone microarchitecture, through novel grey-level texture measurements on lumbar spine DXA images appears to be normal in hypoparathyroidism [37].

Peripheral quantitative computed tomography (pQCT) and high-resolution peripheral quantitative computed tomography (HRpQCT), as well as direct histomorphometric analysis of bone by transiliac bone biopsy, show that both cortical and trabecular compartments of bone are affected in these patients, including increased cortical volumetric BMD and trabecular bone volume fraction and decreased cortical porosity [31]. By pQCT of the radius, volumetric BMD at cancellous and cortical compartments, as well as cortical area and thickness, result greater in hypoparathyroid patients compared to controls [37]. In hypoparathyroid subjects, the use of HRpQCT confirmed the increase in cortical volumetric BMD but showed reduced cortical thickness, with lower cortical porosity. Trabecule were more numerous but thinner in these patients [37]. Lastly, in another study, percutaneous iliac crest bone biopsies of 25 subjects with hypoparathyroidism, and 25 controls were analyzed by high-resolution microcomputed tomography. Hypoparathyroid subjects had increased cancellous bone volume, trabecular thickness, number and connectivity. Therefore, it was confirmed that cancellous bone was 
markedly abnormal in hypoparathyroidism, suggesting that PTH is required to maintain normal trabecular structure [38]. Until now, as already mentioned, it is still unclear whether these structural alterations and the dynamic skeletal abnormalities may affect bone strength or fracture risk in hypoparathyroid patients [37].

Bone turnover markers in chronic hypoparathyroidism treated with conventional treatment (calcium and vitamin D) are usually in the lower half of the normal range [33,38-40]. The low bone turnover in hypoparathyroidism has been shown by histomorphometric assessment of the transiliac bone biopsy [33,39,41], to be associated with a positive bone balance [42]. A study evaluated bone histomorphometry in hypoparathyroid patients treated with conventional treatment, through the analysis of iliac crest bone biopsies derived from 12 subjects ( 8 women and 4 men) and from 13 ageand gender-matched normal controls [42]. This investigation showed that mean total resorption rate, the resorption depth, and fractional formation surface were reduced, and the resorption period was prolonged. Lastly, the quiescent period was prolonged and the activation frequency was reduced. In the absence of PTH, vitamin D alone was not able to normalize bone resorption and bone turnover in patients with hypoparathyroidism [42].

\section{Fracture risk}

In hypoparathyroidism, the skeletal features including low bone remodeling and high BMD, could suggest that bone is hypermature, with an altered bone quality and, therefore, at higher risk of fracture compared to eu-parathyroid bone [5]. However, hypoparathyroidism is a rare disorder, few data are published regarding fracture risk in these patients, and the results are conflicting [5].

The Danish case-control survey with 688 patients with postsurgical hypoparathyroidism (due to non-malignant causes treated with standard treatment for more than 6 months) showed that hypoparathyroid patients did not have an increased risk of long-term overall fractures compared with controls, and the risk of fractures at the upper extremities was significantly decreased in patients with hypoparathyroidism [32]. On the other hand, in another Danish investigation with 180 subjects with nonsurgical hypoparathyroidism, hypoparathyroid patients had a significantly increased risk of fractures at the upper extremities, although the overall fracture risk was similar between cases and controls [29]. A small study evaluated 33 postmenopausal women underwent total thyroidectomy due to thyroid cancer, among them, 13 subjects became hypoparathyroid. The incidence of spinal deformity (assessed by spinal radiographs), was significantly lower in women with hypoparathyroidism than in controls, and the age-matched BMD was clearly higher [43]. On the other hand, a small study described that in 16 patients with hypoparathyroidism had an increased frequency of morphometric vertebral fractures, compared to 17 age- and body mass index (BMI) matched normal controls, without no significant differences in lumbar spine or total hip BMD between groups [44]. Lastly, a study evaluated vertebral fractures, assessed by morphometry, in 104 patients affected by idiopathic hypoparathyroidism and 64 healthy controls [45]. The vertebral fractures were described in $18.3 \%$ of patients with idiopathic hypoparathyroidism and $4.7 \%$ of controls. Mean BMD at lumbar spine and hip were higher by $21.4 \%$ and $8.6 \%$, respectively, in idiopathic hypoparathyroidism than in controls $(\mathrm{P}<0.001)$, therefore showing that, despite the increased BMD, the prevalence of vertebral-fractures was greater in patients with idiopathic hypoparathyroidism (especially in postmenopausal women and those on anticonvulsant therapy) [45].

\section{Clinical manifestations of nervous system}

\section{Central nervous system}

Hypoparathyroid patients can manifest focal or generalized seizures, tonic-clonic type, caused by severe hypocalcaemia [8]. Recently, some studies have reported a $3.8 \%-8 \%$ prevalence of seizures in patients with hypoparathyroidism [26,28].

Basal ganglia calcification (BGC) is a well-known clinical complication of hypoparathyroidism [46-49]. The reported prevalence of BGC in hypoparathyroidism varies between $52 \%$ and $74 \%$ [26,49], whereas in the general population the prevalence of BGC is not well established, but estimates are significantly lower (2-12.5\%) [26,47]. In a cohort of 25 patients with CaSR mutations, 36\% had BGC 
[26,48]. In contrast, in a cohort of 145 patients affected by nonsurgical hypoparathyroidism $74 \%$ had BGC [49]. However, in neither of these investigations there was a systematic screening for BGC [26]. The most common site of the central nervous system calcifications is the basal ganglia. However, these can occur also in the grey and white matter junction, the cerebellar parenchyma, the thalamus and the dentate nucleus. The occurrence of BGC and its progression have been associated with low calcium/ phosphorus ratio and long-standing duration of hypocalcaemic symptoms, even though, the exact pathogenesis of these calcifications is not well known [49]. The abnormal phosphate homeostasis seems to have a role in extraskeletal calcifications in these patients [8]. In this regard, two genes, such as sodium-dependent phosphate transporter 2 (PIT2) and xenotropic and polytropic retrovirus receptor 1 (XPR1) encoding proteins involved in phosphate transport have been associated with familial idiopathic basal ganglia calcification [50,51]. The possible clinical consequences of the central nervous system calcifications are not clear. Neurological abnormalities and cognitive deficits may be pathophysiologically related to the presence of intracranial calcification in this disorder, but the relationship is unknown. Parkinsonism and dystonia have been described in chronic hypoparathyroidism, but with a prevalence lower than that of BGC [26,49]. Lastly, a mutation in a type III sodium-phosphate transporter leading to impaired cellular uptake of inorganic phosphate was reported to be a cause of familial idiopathic BGC, suggesting that extracellular accumulation of phosphate in the setting of chronic hyperphosphatemia may contribute to BGC in hypoparathyroidism [26,51].

\section{Peripheral nervous system}

Hypocalcaemia associated to hypoparathyroidism can cause neuromuscular irritability, because partially depolarizes the resting membrane potential of a neuron, stimulating the triggering action potentials [52]. Paresthesia in the extremities and in the peri-oral and oral area are the manifestations of sensory neuron irritability, as well as muscle spasms or tetany of motor neuron irritability. Muscle spasms or tetany can vary from the carpopedal spasm, characterized by spasmic muscle contractions of the forearm, hand, lower leg and/or feet, to life-threatening laryngospasm [53,54].

The Chvostek sign and Trousseau sign can detect an increased neuromuscular irritability [8,55]. Chvostek's sign is elicited by tapping the facial nerve in front of the ear, inducing the facial muscles on the ipsilateral side to twitch. This causes contractions ranging from the upper lip and nose to the entire half-face when severe and significant hypocalcemia is present. Trousseau's sign is elicited by placing a blood pressure cuff around the arm and inflating the cuff to greater than the systolic pressure and holding this in place for 3 min (occluding the brachial artery). The absence of blood flow and hyperexcitability of the muscles caused by hypocalcemia determines a painful flexion of the wrist and metacarpophalangeal joints, with extension of the distal interphalangeal and proximal interphalangeal joints, and abduction of the fingers [56].

\section{Neuropsychiatric manifestations}

The Danish case-control survey, conducted on patients with postsurgical hypoparathyroidism, showed that hypoparathyroid patients had a significantly increased risk of hospitalization due depression/bipolar affective disorders (HR 1.99, 95\% CI 1.14-3.46) compared with age-matched and sex-matched controls [32]. Therefore, increased risk of depression and other types of neuropsychiatric disorders seems to be associated to postsurgical hypoparathyroidism.

An other Danish case-control study described that also patients with nonsurgical hypoparathyroidism had a significantly increased risk of neuropsychiatric complications (HR 2.45), compared with controls [29]. However, further studies are needed.

\section{Ophthalmological manifestations}

Cataract is a typical manifestation of hypoparathyroidism. In a case-control study, the patients with idiopathic hypoparathyroidism and cataracts, were significantly younger than patients with cataracts without hypoparathyroidism and had evidence of more-severe posterior capsule disease and a higher rate of anterior capsule disease [57]. In the Danish case-control investigations, patients affected by 
non-surgical hypoparathyroidism had an increased hazard ratio of 4.21 (95\% CI: $2.13-8.34$ ) compared with controls, but the risk of cataracts in patients with post-surgical hypoparathyroidism was not significantly different compared to the general population. This studies suggest that age of onset and/or duration of hypoparathyroidism are important contributing factors $[8,28,29]$. The etiology of the cataract formation is not well known in these patients. However, some preclinical investigations hypothesize that it may be due to chronic low serum calcium levels [58]. Recent studies have shown an increased risk of posterior subcapsular cataracts, likely due to elevated calcium $\times$ phosphorus product occurring in lenses in the eyes [56]. Lastly, papilledema has also be described in hypoparathyroidism and tends to improve with normalization of serum calcium levels [8].

\section{Cardiovascular manifestations}

Cardiac arrhythmias can occur in patients with hypoparathyroidism in the presence of hypocalcaemia, but the treatment of hypocalcaemia usually resolve promptly these symptoms [59,60]. The most frequent ECG alteration is the prolongation of the QTc interval, due to an increase in phase 2 of the action potential, along with prominent $U$ wave and $T$ wave abnormalities [59]. Moreover, in some studies, dilated cardiomyopathy due to hypocalcemia affecting the cardiac function, albeit rarely, has been reported [61-63]. Recently, in a cohort study of 120 patients with hypoparathyroidism, only one patient had dilated cardiomyopathy attributed to chronic hypocalcemia [26]. Usually the improvement of cardiac dysfunction occurs with normalization of hypocalcemia. However, a case report of a pediatric patient with severe hypocalcaemia reported a non-reversible component in hypocalcaemia-associated dilated cardiomyopathy [61]. Overall, compared with controls, the Danish case-control study showed that patients with postsurgical hypoparathyroidism had no increased risk of cardiac arrhythmias (HR, 1.11; 95\% CI, 0.79-1.57) or cardiovascular disease or death (HR, 0.89; 95\% CI, 0.73-1.09) [28].

\section{Muscular manifestations}

Myopathy is a rare manifestation of hypoparathyroidism [64]. Only a small number of reports regarding hypocalcemic myopathy due to hypoparathyroidism have been reported [64]. A retrospective study on 9 patients affected by idiopathic hypoparathyroidism found an inverse relationship between serum calcium and creatine phosphokinase [65]. Mild to moderate muscle cell degeneration was present in almost all patients, and the severity of changes in muscles was related to the duration of hypocalcemia and not only to its degree [65]. Lastly, a cross-sectional study design showed that muscle strength and maximal force production was significantly reduced in hypoparathyroid patients compared with age matched and sex-matched controls, while the time for performing the Timed Up \& Go test and the repeated Chair stands test was significantly longer than in the hypoparathyroidism group and the control group [66].

\section{Dental manifestations}

Dental aberrations have been described in non-surgical hypoparathyroidism with symptoms including shortened roots, hypoplastic enamel and hypoplastic or absent teeth [67-69]. However, in the future, further studies should be conducted for better characterizing this complication [69].

\section{Dermatological manifestations}

Hypoparathyroidism can cause also some skin manifestations. Regarding this specific clinical manifestation, an observational study was conducted on 21 patients mostly with postsurgical hypoparathyroidism [70]. Mucocutaneous manifestations were described in $76 \%$ of subjects, among these, the most common mucocutaneous features was found in the hairs, including the loss of axillary hair, loss of pubic hair, coarsening of body hair, and alopecia areata. Brittle and ridged nail, followed by onycholysis, onychosezia, and onychomadesis were reported. The most common skin manifestation were xerotic skin in 11 patients (52.38\%), followed by pellagra-like skin pigmentation, pustular psoriasis and acne form eruption, bullous impetigo, etc. Only one case of oral candidiasis was described [70]. In several case 
reports was documented the generalized pustular psoriasis associated with pus-filled blisters, associated with severe hypocalcaemia. This clinical manifestation improved with treatment [71,72].

\section{Quality of life of chronic hypoparathyroidism}

Several studies have assessed the quality of life in hypoparathyroidism and have showed that hypoparathyroid patients reported reduced quality of life compared to either a normal population or suitable controls $[2,3,32,73-77]$. These investigations described that hypoparathyroid patients often complain about fatigue, muscle spasms, pain, and paresthesia, cognitive symptoms including "brain fog" and inability to concentrate, anxiety and tendency to depression $[2,3]$. The etiology and the duration of the hypoparathyroidism seem to be irrespective from reduced quality of life [5].

A German cross-sectional, controlled study, carried out with 25 women affected by postsurgical hypoparathyroidism compared to 25 controls (with a history of thyroid surgery but intact parathyroid function), reported that hypoparathyroid patients had significantly higher global complaint scores with predominant increases in the subscale scores for anxiety, phobic anxiety and their physical equivalents [73].

An American (United States) study tested the hypothesis that surgeons and their patients could underestimate the potential negative impact of postsurgical permanent hypoparathyroidism on quality of life [78]. A total of 340 postsurgical patients with permanent hypoparathyroidism, 200 controls, and 102 surgeons were included in this investigation. Forty-seven percent of hypoparathyroid patients reported that their health was "much worse" than before surgery, reporting of note interference with social activities, paresthesias, muscle cramping, and medications. On the other hand, surgeons and controls underestimated the negative impact of hypoparathyroidism on quality of life compared to patients with chronic hypoparathyroidism [78]. Moreover, in United States, a web-based instrumentconducted survey of 374 patients with hypoparathyroidism showed that hypoparathyroid patients had a high burden of illness and experience a broad spectrum of symptoms, with a multidimensional impact on their lives [74].

An Indian cross-sectional study showed that 62 patients with idiopathic hypoparathyroidism had a higher proportion of neuropsychiatric and cognitive dysfunction and decreased quality of life compared to controls [79].

Additional data on quality of life in these patients are obtained by trials with PTH treatments. In a trail, 69 hypoparathyroid patients at baseline completed the RAND 36-Item Short Form (SF-36) Health Survey, and scored significantly lower than the normative reference range in all 8 domains [75]. In addition, another randomized controlled trial conducted in 62 patients with chronic hypoparathyroidism reported that at baseline the SF-36 questionnaire showed a significantly lower baseline score, compared to the norm-based population, at most subscales. At baseline, there were no correlations between SF-36 measurements and BMI, age, duration of disease, or etiology of disease [76].

Lastly, a cohort study with patients affected by nonsurgical hypoparathyroidism (57 patients) and PHP (30 patients) reported that quality of life, evaluated with SF-36 and WHO-5 Well Being Index, was impaired equally among patients with nonsurgical hypoparathyroidism and PHP [80].

\section{Other clinical manifestations}

Many syndromic and nonsyndromic forms of hypoparathyroidism can include a broad spectrum of signs and symptoms (see review regarding causes and pathophysiology of hypoparathyroidism), such as dysmorphism, short stature, immunodeficiency, cardiac anomalies, skeletal abnormalities, hearing loss, renal anomalies, and many others [1].

\section{Pregnancy and breastfeeding}

Though PTH has a negligible role in regulating calcium homeostasis in pregnancy and lactation in women without hypoparathyroidism, in case of women with chronic hypoparathyroidism treated with calcium and vitamin D metabolites or analogs supplement are considered at considerable risk for serum calcium oscillations (hypercalcemia and hypocalcaemia) when pregnant or nursing [10]. An 
inadequate control of calcium and phosphate homeostasis during pregnancy can cause miscarriage, stillbirth, premature labor and even neonatal death [10,81-83]. Moreover, maternal hypocalcaemia can impair neonatal bone development and cause compensatory hyperparathyroidism in the newborn, as well as, fractures, skeletal deformities, parathyroid hyperplasia and clinical complications, including respiratory distress, poor feeding and hypotonia may occur [10]. On the other hand, maternal hypercalcemia, due to high calcium/vitamin D supplement dosage, can suppress fetal parathyroid development causing neonatal hypocalcaemia. Therefore, pregnant and nursing women with hypoparathyroidism should be monitored in order to carefully adjust the therapy [10].

\section{Diagnosis and clinical evaluation}

The biochemical diagnosis of hypoparathyroidism is based on the measurement of albumincorrected or ionized serum calcium below the lower limits of the normal range and low, undetectable or inappropriate levels of PTH (measured by either a second- or third-generation immunoassay) on at least two occasions separated by at least 2 weeks, after hypomagnesemia has been ruled out $[2,5,10]$. If serum magnesium deficiency is detected, it is useful to measure the 24 -h urinary magnesium level before repletion is initiated [2]. Serum phosphorus concentrations are usually high or at the upper limit of the normal range [2]. Moreover, biochemical evaluation should be extended to the measurements of $25 \mathrm{OHD}, 1,25(\mathrm{OH})_{2} \mathrm{D}, 24$-h urinary calcium excretion, estimated or calculated GFR, and eventually biochemical stone risk profile, if necessary [5,56]. In case of hypoparathyroidism after anterior neck surgery, chronic hypoparathyroidism can be diagnosed 6 months after surgery [5].

The personal medical history should be collected in these patients, including history of anterior neck surgery, gastrointestinal, renal, and skeletal symptoms, general quality of life, and treatments, as well as family history [5]. Indeed, review of the patient's medical and family histories may suggest the etiology of hypoparathyroidism, such as a personal history of neck surgery suggests that parathyroid function may have been compromised by the surgical procedure, and a family history of hypocalcemia can suggest a genetic cause [2]. The presence of other autoimmune endocrinopathies, such as adrenal insufficiency or candidiasis can assume autoimmune polyendocrine syndrome type 1 , or immunodeficiency and other congenital defects point to the DiGeorge syndrome [2]. In a patient with hypoparathyroidism of unknown etiology or if the patient's presentation suggests a genetic basis (i.e. young age, family history, multiple autoimmune features) should be considered genetic counseling, genetic testing and/or family screening [5,10].

Physical examination should be performed, focusing on: anterior neck (for signs of previous surgery), signs of neuromuscular irritability, using Chvostek's, Trousseau's sign, eye examination (for cataracts and calcifications), nail beds examination (for fungal infection), range of motion of joints evaluation, skin manifestations such as mucosal candidiasis, vitiligo, and generalized bronzing and signs of liver disease (suggestive of hemochromatosis, or other causes of iron overload) [2,5]. Clinical features, including growth failure, congenital anomalies, hearing loss, or retardation suggest the possibility of genetic disease [2].

Lastly, imaging studies should be performed, such as skull x-ray for basal ganglia and other intracerebral calcifications, abdomen ultrasound for renal stones and calcifications, and dual-energy x-ray absorptiometry for BMD measurement [5].

\section{Clinical monitoring}

Biochemical monitoring of serum ionized or albumin adjusted total calcium, phosphate, magnesium, creatinine levels, and eGFR, in addition to assessment of symptoms due to hypocalcaemia and hypercalcemia at regular time intervals is recommended $[5,10]$. The frequency of biochemical monitoring depends by the extent to which a patient is stable on a given dosing regimen. In case of patients adequately controlled, the monitoring could be performed on a yearly or twice-yearly basis, in other cases the patients could require much more frequent monitoring [5]. When the dosage of calcium and active vitamin $\mathrm{D}$ supplementations are adjusted, more frequent monitoring is suggested, also several times per week, until a stable serum calcium concentration is documented [5]. Renal function is monitored annually by a 24-h urine collection for calcium and creatinine excretion along with a 
measured creatinine clearance or eGFR. The frequency of monitoring might be longer in case of stability of renal function over time, and not associated with renal complications. The measurement of urinary magnesium excretion can be helpful in certain situations [5]. In case of symptoms due to renal stones or increase of serum creatinine levels renal imaging is recommended [10]. In asymptomatic patients with a history of renal lithiasis or nephrocalcinosis, renal imaging is recommended every 5 years [5].

Frequent monitoring of patients with autosomal dominant hypocalcaemia (ADH), treated with standard treatment, is recommended because they may be at high risk of hypercalciuria and renal complications [10].

It is not known whether monitoring of central nervous system calcifications detected at baseline screening is useful, since no clinical features are attributed to these calcifications $[5,26,49]$.

Eyes examination for cortical cataracts evaluation depends on the specific clinical situation [5].

BMD monitoring also in hypoparathyroidism is recommended as per recommendations of the International Society of Clinical Densitometry [5,84].

It is suggested monitoring serum ionized calcium regularly, every 2-3 weeks, during pregnancy and breastfeeding, in women treated with calcium and activated vitamin D analogs supplements [10]. Lastly, the pediatrician and/or neonatologist should be informed of maternal hypoparathyroidism [10].

\section{Summary}

Hypoparathyroidism is a rare endocrine disease, and, as a consequence. It is a less studied pathology compared to other common endocrine disorders. However, recently, some studies have been focused on this disorder, including its clinical manifestations. The long-term consequences of chronic hypoparathyroidism can be severe and may require the intervention of several specialists, given the wide spectrum of clinical complications that can occur. The most severe long-term complications are nephrocalcinosis and renal failure, markedly increased in hypoparathyroid patients treated with chronic conventional treatment. Therefore, an adequate monitoring of renal function should be performed. Moreover, an increased risk of seizures and calcification of the basal ganglia is clearly documented. However, further studies should be carried out on the pathogenesis of calcifications of the central nervous system and their clinical consequences. Regarding bone status, patients with chronic hypoparathyroidism treated with calcium and vitamin D have higher BMD and lower bone turnover compared to general population. Currently it is unclear if this impairs bone strength and can increase risk of fragility fractures. A reduction of quality of life in hypoparathyroid patients treated with standard treatment has been described in several studies, but, in the future, it will be necessary to clarify whether PTH deficiency has a role in determining central nervous system alterations. Lastly, in the future, further studies will have to be conducted in order to clarify the pathogenesis and the epidemiology of the clinical manifestations, and of the long-term complications associated with hypoparathyroidism, both in patients treated with conventional treatment and replacement therapy with recombinant human parathyroid hormone [rhPTH (1-84)].

\section{Practice points}

- The diagnosis of hypoparathyroidism is based on: hypocalcemia (albumin-corrected or ionized serum calcium), undetectable or inappropriate levels of PTH on (at least two occasions separated by at least 2 weeks). Serum phosphate levels are usually in the upper normal or markedly elevated range (helpful but not mandatory).

- Personal and family medical history is useful to identify the cause of hypoparathyroidism.

- Genetic testing and/or family screening should be performed in case of hypoparathyroidism of unknown etiology or suspicion of genetic basis.

- Physical examination should be performed to evaluate all possible complications.

- Biochemical assessment (bone metabolism, renal function) and target organ imaging [X-ray (skull), renal ultrasound or computed tomography scanning and BMD by dual-energy $x$-ray absorptiometry] should be performed based on the patient's clinical situation. 


\section{Research agenda}

Regarding the clinical manifestations of chronic hypoparathyroidism, further studies are necessary in the following issues:

- Pathogenesis of ectopic calcifications, and clinical consequences of brain calcifications.

- Bone quality/strength and risk of fragility fractures.

- Specific validated questionnaires for the evaluation of quality of life.

- Natural history and comorbidity.

\section{Funding source}

None.

\section{Conflicts of interest}

Brandi ML: is a consultant for Alexion, Bruno Farmaceutici, Shire, Servier, Kyowa Kirin; academic grants and/or speaker: Abiogen, Alexion, Amgen, Bruno Farmaceutici, Eli Lilly, Kyowa Kirin, MSD, NPS, Servier, Shire, SPA; she has received honoraria from: Amgen, Bruno Farmaceutici, Kyowa Kirin. The other authors declare that they have no conflict of interests.

\section{References}

*[1] Shoback DM, Bilezikian JP, Costa AG, et al. Presentation of hypoparathyroidism: etiologies and clinical features. J Clin Endocrinol Metab 2016;101:2300-12.

*[2] Shoback D. Clinical practice. Hypoparathyroidism. N Engl J Med 2008;359:391-403.

*[3] Bilezikian JP, Khan A, Potts Jr JT, et al. Hypoparathyroidism in the adult: epidemiology, diagnosis, pathophysiology, targetorgan involvement, treatment, and challenges for future research. J Bone Miner Res 2011;26:2317-37.

[4] De Sanctis V, Soliman A, Fiscina B. Hypoparathyroidism: from diagnosis to treatment. Curr Opin Endocrinol Diabetes Obes 2012;19:435-42.

*[5] Brandi ML, Bilezikian JP, Shoback D, et al. Management of hypoparathyroidism: summary statement and guidelines. J Clin Endocrinol Metab 2016:101:2273-83.

*[6] Mannstadt MM, Mitchell DM. Clinical manifestations of hypoparathyroidism. In: Bilezikian JP, Marcus R, Levine MA, et al., editors. The parathyroids. London: Academic Press; 2015. p. 761-70.

[7] Goswami R, Millo T, Mishra S, et al. Expression of osteogenic molecules in the caudate nucleus and gray matter and their potential relevance for basal ganglia calcification in hypoparathyroidism. J Clin Endocrinol Metab 2014;99:1741-8.

*[8] Mannstadt M, Bilezikian JP, Thakker RV, et al. Hypoparathyroidism. Nat Rev Dis Prim 2017;5:17080.

[9] Astor MC, Løvås K, Debowska A, et al. Epidemiology and health-related quality of life in hypoparathyroidism in Norway. J Clin Endocrinol Metab 2016;101:3045-53.

*[10] Bollerslev J, Rejnmark L, Marcocci C, et al. European society of endocrinology clinical guideline: treatment of chronic hypoparathyroidism in adults. Eur J Endocrinol 2015;173:G1-20.

[11] Holt EH, Bilezikian JP. Calcium disorders. In: McKean SC, Ross JJ, Dressler DD, et al., editors. Principles and practice of hospital medicine. New York, NY: McGraw Hill; 2012. p. 2067-76.

[12] Mantovani G, Bastepe M, Monk D, et al. Diagnosis and management of pseudohypoparathyroidism and related disorders: first international Consensus Statement. Nat Rev Endocrinol 2018;14:476-500.

[13] Segre BV, D'Amour P, Potts JT. Metabolism of radioiodinated bovine parathyroid hormone in the rat. Endocrinology 1976; 99:1645-52.

[14] Zhang CX, Weber BV, Thammavong J, et al. Identification of carboxylterminal peptide fragments of parathyroid hormone in human plasma at low-picomolar levels by mass spectrometry. Anal Chem 2006;78:1636-43.

[15] Logue FC, Perry B, Chapman RS, et al. A two-site immunoradiometric assay for PTH(1-84) using N and C terminal specific monoclonal antibodies. Ann Clin Biochem 1991;28:160-6.

[16] Thakker RV, Bringhurst FR, Juppner H. Genetic disorders of calcium homeostasis caused by abnormal regulation of parathyroid hormone secretion or responsiveness. In: DeGroot LJ, Jameson JL, editors. Endocrinology. Philadelphia, PA: W. B. Saunders Company; 2016. p. 1063-89.

[17] Eastell R, Brandi ML, Costa AG, et al. Diagnosis of asymptomatic primary hyperparathyroidism: proceedings of the Fourth International Workshop. J Clin Endocrinol Metab 2014;99:3570-9.

[18] Nussbaum SR, Zahradnik RJ, Lavigne JR, et al. Highly sensitive two-site immunoradiometric assay of parathyrin, and its clinical utility in evaluating patients with hypercalcemia. Clin Chem 1987;33:1364-7.

[19] John MR, Goodman WG, Gao P, et al. A novel immunoradiometric assay detects full-length human PTH but not aminoterminally truncated fragments: implications for PTH measurements in renal failure. J Clin Endocrinol Metab 1999;84: 4287-90. 
[20] Inaba M, Nakatsuka K, Imanishi Y, et al. Technical and clinical characterization of the Bio-PTH (1-84) immunochemiluminometric assay and comparison with a second-generation assay for parathyroid hormone. Clin Chem 2004;50: 385-90.

[21] D'Amour P, Brossard JH, Räkel A, et al. Evidence that the amino-terminal composition of non-(1-84) parathyroid hormone fragments starts before position 19. Clin Chem 2005;51:169-76.

[22] Steen O, Khan A. Role of magnesium in parathyroid physiology. In: Brandi ML, Brown EM, editors. Hypoparathyroidism. Milan, Italy: Springer-Verlag Italia; 2015. p. 61-7.

[23] Rizzoli R. Hypoparathyroidism during magnesium deficiency or excess. In: Brandi ML, Brown EM, editors. Hypoparathyroidism. Milan, Italy: Springer-Verlag Italia; 2015. p. 397-403.

[24] Bilezikian JP, Brandi ML, Cusano NE, et al. Management of hypoparathyroidism: present and future. J Clin Endocrinol Metab 2016;101:2313-24.

[25] Hobaus J, Thiem U, Hummel DM, et al. Role of calcium, vitamin D, and the extrarenal vitamin D hydroxylases in carcinogenesis. Anti Cancer Agents Med Chem 2013;13:20-35.

[26] Mitchell DM, Regan S, Cooley MR, et al. Long-term follow-up of patients with hypoparathyroidism. J Clin Endocrinol Metab 2012;97:4507-14.

[27] Levy I, Licht C, Daneman A, et al. The impact of hypoparathyroidism treatment on the kidney in children: long-term retrospective follow-up study. J Clin Endocrinol Metab 2015;100:4106-13.

*[28] Underbjerg L, Sikjaer T, Mosekilde L, et al. Cardiovascular and renal complications to postsurgical hypoparathyroidism: a Danish nationwide controlled historic follow-up study. J Bone Miner Res 2013;28:2277-85.

*[29] Underbjerg L, Sikjaer T, Mosekilde L, et al. The epidemiology of nonsurgical hypoparathyroidism in Denmark: a nationwide case finding study. J Bone Miner Res 2015;30:1738-44.

[30] Silva B, Kousteni S. Cellular actions of PTH: osteoblasts, osteoclasts, and osteocytes. In: Bilezikian J, editor. The parathyroids. 3nd ed. Elsevier Inc.; 2015. p. 127-37.

[31] Cusano NE, Nishiyama KK, Zhang C, et al. Noninvasive assessment of skeletal microstructure and estimated bone strength in hypoparathyroidism. J Bone Miner Res 2016;31:308-16.

*[32] Underbjerg L, Sikjaer T, Mosekilde L, et al. Postsurgical hypoparathyroidism-risk of fractures, psychiatric diseases, cancer, cataract, and infections. J Bone Miner Res 2014;29:2504-10.

[33] Rubin MR, Dempster DW, Zhou H, et al. Dynamic and structural properties of the skeleton in hypoparathyroidism. J Bone Miner Res 2008;23:2018-24.

[34] Sikjaer T, Rejnmark L, Rolighed L, et al. The effect of adding PTH(1-84) to conventional treatment of hypoparathyroidism: a randomized, placebo-controlled study. J Bone Miner Res 2011:26:2358-70.

[35] Cusano NE, Rubin MR, McMahon DJ, et al. Therapy of hypoparathyroidism with PTH(1-84): a prospective four-year investigation of efficacy and safety. J Clin Endocrinol Metab 2013;98:137-44.

[36] Winer KK, Sinaii N, Reynolds J, et al. Long-term treatment of 12 children with chronic hypoparathyroidism: a randomized trial comparing synthetic human parathyroid hormone 1-34 versus calcitriol and calcium. J Clin Endocrinol Metab 2010; 95:2680-8.

[37] Silva BC, Rubin MR, Cusano NE, et al. Bone imaging in hypoparathyroidism. Osteoporos Int 2017;28:463-71.

[38] Rubin MR, Dempster DW, Kohler T, et al. Three dimensional cancellous bone structure in hypoparathyroidism. Bone 2010; $46: 190-5$

[39] Rubin MR, Dempster DW, Sliney Jr J, et al. PTH(1-84) administration reverses abnormal bone-remodeling dynamics and structure in hypoparathyroidism. J Bone Miner Res 2011;26:2727-36.

[40] Marcucci G, Cianferotti L, Parri S, et al. HypoparaNet: a database of chronic hypoparathyroidism based on expert medicalsurgical centers in Italy. Calcif Tissue Int 2018;103:151-63.

[41] Dempster DW. Bone histomorphometry in hypoparathyroidism. In: Brandi ML, Brown EM, editors. Hypoparathyroidism. New York, NY: Springer; 2015. p. 287-96.

[42] Langdahl BL, Mortensen L, Vesterby A, et al. Bone histomorphometry in hypoparathyroid patients treated with vitamin D. Bone 1996;18:103-8.

[43] Fujiyama K, Kiriyama T, Ito M, et al. Attenuation of postmenopausal high turnover bone loss in patients with hypoparathyroidism. J Clin Endocrinol Metab 1995;80:2135-8.

[44] Mendonça ML, Pereira FA, Nogueira-Barbosa MH, et al. Increased vertebral morphometric fracture in patients with postsurgical hypoparathyroidism despite normal bone mineral density. BMC Endocr Disord 2013;3. 13-1.

[45] Chawla H, Saha S, Kandasamy D, et al. Vertebral fractures and bone mineral density in patients with idiopathic hypoparathyroidism on long-term follow-up. J Clin Endocrinol Metab 2017:102:251-8.

[46] Eaton LM, Camp JD, Love JG. Symmetric cerebral calcification, particularly of the basal ganglia, demonstrable roentgenographically. Arch Neurol Psychiatry 1939;41:921-42.

[47] Fénelon G, Gray F, Paillard F, et al. A prospective study of patients with CT detected pallidal calcifications. J Neurol Neurosurg Psychiatry 1993;56:622-5.

[48] Raue F, Pichl J, Dorr HG, et al. Activating mutations in the calcium-sensing receptor: genetic and clinical spectrum in 25 patients with autosomal dominant hypocalcaemia: a German survey. Clin Endocrinol (Oxf) 2011;75:760-5.

[49] Goswami R, Sharma R, Sreenivas V, et al. Prevalence and progression of basal ganglia calcification and its pathogenic mechanism in patients with idiopathic hypoparathyroidism. Clin Endocrinol (Oxf) 2012;77:200-6.

[50] Legati A, Giovannini D, Nicolas G, et al. Mutations in XPR1 cause primary familial brain calcification associated with altered phosphate export. Nat Genet 2015;47:579-81.

[51] Wang C, Li Y, Shi L, et al. Mutations in SLC20A2 link familial idiopathic basal ganglia calcification with phosphate homeostasis. Nat Genet 2012;44:254-6.

[52] Frankenhaeuser B, Hodgkin AL. The action of calcium on the electrical properties of squid axons. J Physiol 1957;137: $218-44$.

[53] Williams GT, Brown M. Laryngospasm in hypoparathyroidism. J Laryngol Otol 1974;88:369-73.

[54] Chou CT, Siegel B, Mehta D. Stridor and apnea as the initial presentation of primary hypoparathyroidism. Int J Pediatr Otorhinolaryngol 2016;80:30-2. 
[55] Jesus JE, Landry A. Images in clinical medicine. Chvostek's and Trousseau's signs. N Engl J Med 2012 13;367:e15.

[56] Abate EG, Clarke BL. Review of hypoparathyroidism. Front Endocrinol (Lausanne) 2017 16;7:172.

[57] Saha S, Gantyala SP, Aggarwal S, et al. Long-term outcome of cataract surgery in patients with idiopathic hypoparathyroidism and its relationship with their calcemic status. J Bone Miner Metab 2017;35:405-11.

[58] Bunce GE, Kinoshita J, Horwitz J. Nutritional factors in cataract. Annu Rev Nutr 1990;10:233-54.

[59] Vered I, Vered Z, Perez JE, et al. Normal left ventricular performance documented by Doppler echocardiography in patients with long-standing hypocalcemia. Am J Med 1989;86:413-6.

[60] Newman DB, Fidahussein SS, Kashiwagi DT, et al. Reversible cardiac dysfunction associated with hypocalcemia: a systematic review and meta-analysis of individual patient data. Heart Fail Rev 2014;19:199-205.

[61] Velayuthan S, Gungor N, McVie R. Hypocalcemic cardiomyopathy as initial presentation of primary hypoparathyroidism. Pediatr Int 2014;56:e23-5.

[62] Tziomalos K, Kakavas N, Kountana E, et al. Reversible dilated hypocalcaemic cardiomyopathy in a patient with primary hypoparathyroidism. Clin Endocrinol (Oxf) 2006;64:717-8.

[63] Avsar A, Dogan A, Tavli T. A rare cause of reversible dilated cardiomyopathy: hypocalcemia. Echocardiography 2004;21: 609-12.

[64] Policepatil SM, Caplan RH, Dolan M. Hypocalcemic myopathy secondary to hypoparathyroidism. WMJ 2012;111:173-5.

[65] Dai CL, Sun ZJ, Zhang X, et al. Elevated muscle enzymes and muscle biopsy in idiopathic hypoparathyroidism patients. J Endocrinol Invest 2012;35:286-9.

[66] Sikjaer T, Moser E, Rolighed L, et al. Concurrent hypoparathyroidism is associated with impaired physical function and quality of life in hypothyroidism. J Bone Miner Res 2016;31:1440-8.

[67] Jensen SB, Illum F, Dupont E. Nature and frequency of dental changes in idiopathic hypoparathyroidism and pseudohypoparathyroidism. Scand J Dental Res 1981;89:26-37.

[68] Srirangarajan S, Satyanarayan A, Ravindra S, et al. Dental manifestation of primary idiopathic hypoparathyroidism. J Indian Soc Periodontol 2014;18:524-6.

[69] Hejlesen J, Underbjerg L, Gjørup H, et al. Dental findings in patients with non-surgical hypoparathyroidism and pseudohypoparathyroidism: a systematic review. Front Physiol 2018;19(9):701.

[70] Sarkar S, Mondal M, Das K, et al. Mucocutaneous manifestations of acquired hypoparathyroidism: an observational study. Indian J Endocrinol Metab 2012;16:819-20.

[71] Lee Y, Nam YH, Lee JH, et al. Hypocalcaemia-induced pustular psoriasis-like skin eruption. Br J Dermatol 2005;152:591-3.

[72] Guerreiro de Moura CA, de Assis LH, Góes P, et al. A case of acute generalized pustular psoriasis of von Zumbusch triggered by hypocalcemia. Case Rep Dermatol 2015;7:345-51.

[73] Arlt W, Fremerey C, Callies F, et al. Well-being, mood and calcium homeostasis in patients with hypoparathyroidism receiving standard treatment with calcium and vitamin D. Eur J Endocrinol 2002;146:215-22.

[74] Hadker N, Egan J, Sanders J, et al. Understanding the burden of illness associated with hypoparathyroidism reported among patients in the paradox study. Endocr Pract 2014;20:671-9.

[75] Cusano NE, Rubin MR, McMahon DJ, et al. PTH(1-84) is associated with improved quality of life in hypoparathyroidism through 5 years of therapy. J Clin Endocrinol Metab 2014;99:3694-9.

[76] Sikjaer T, Rolighed L, Hess A, et al. Effects of PTH(1-84) therapy on muscle function and quality of life in hypoparathyroidism: results from a randomized controlled trial. Osteoporos Int 2014;25:1717-26.

[77] Santonati A, Palermo A, Maddaloni E, et al. PTH(1-34) for surgical hypoparathyroidism: a prospective, open-label investigation of efficacy and quality of life. J Clin Endocrinol Metab 2015;100:3590-7.

[78] Cho NL, Moalem J, Chen L, et al. Surgeons and patients disagree on the potential consequences from hypoparathyroidism. Endocr Pract 2014;20:427-46.

[79] Aggarwal S, Kailash S, Sagar R, et al. Neuropsychological dysfunction in idiopathic hypoparathyroidism and its relationship with intracranial calcification and serum total calcium. Eur J Endocrinol 2013;168:895-903.

[80] Cusano NE, Rubin MR, McMahon DJ, et al. The effect of PTH(1-84) on quality of life in hypoparathyroidism. J Clin Endocrinol Metab 2013:98:2356-61.

[81] Mestman JH. Parathyroid disorders of pregnancy. Semin Perinatol 1998;22:485-96.

[82] Callies F, Arlt W, Scholz HJ, et al. Management of hypoparathyroidism during pregnancy - report of twelve cases. Eur J Endocrinol 1998;139:284-9.

[83] Krysiak R, Kobielusz-Gembala I, Okopien B. Hypoparathyroidism in pregnancy. Gynecol Endocrinol 2011;27:529-32.

[84] Schousboe JT, Shepherd JA, Bilezikian JP, et al. Executive summary of the 2013 international society for clinical Densitometry position development conference on bone Densitometry. J Clin Densitom 2013;16:455-66. 